\title{
The effects of water and food deprivation on the heart rate of rats
}

\author{
SACHIO ASHIDA, DEPARTMENT OF PSYCHOLOGY, THE \\ UNIVERSITY OF MICHIGAN, Ann Arbor, Mich. 48104
}

The heart rate (HR) of male hooded rats was recorded in a nonhome cage for three weeks. Then they were deprived of water (Group 1, $N=6$ ) or food (Group 2, $N=6$ ) up to $96 \mathrm{~h}$. HR was recorded at $8 h, 24 h, 48 h, 72 h$, and $96 h$ of deprivation. The HR of Group 1 increased linearly with the duration of water deprivation, while that of Group 2 showed an inverse U-shaped function. These functions suggest that $H R$ is influenced by two concomitant conditions: dehydration and metabolism.

Malmo (1962) suggests that heart rate (HR) as a physiological measure can be applied to determine "appetitionally based drive," and that there should be a functional relationship between HR changes and hours of deprivation. However, HR is an extremely variable measure. For example, while Bélanger \& Feldman (1962) report that their rats showed a monotonic relationship between HR changes and the deprivation of water up to $72 \mathrm{~h}$, Hahn, Stern, \& McDonald (1962) demonstrated that the rats in their first experiment showed no evidence of either the linear function obtained by Bélanger and Feldman or an inverted U-shaped function similar to Hull's model of drive function (1952).

Hufford and Chambers reported, at the Midwestern Psychological Association Meeting in 1963, that HR did not increase as a function of the deprivation of water as reported by Bélanger and Feldman, but it decreased in an apparent linear fashion from about $390 \mathrm{bpm}$ to $350 \mathrm{bpm}$ over a deprivation period extending up to $72 \mathrm{~h}$. In this experiment, Ss were not engaged in operant activities at the time of HR recording. Ducharme (1966) also reports that "... within a normal range, depriving an animal of water cannot per se produce a direct physiological effect leading to an increase in HR and that the conditions of deprivation alone have no appreciable influence on the level of activation as measured by change in HR [p. 103]." Recently Eisman (1966) reported that HR does not vary as a function of water deprivation if confounding factors, such as (a) handling, (b) adaptation to deprivation, and (c) schedules of drinking, are well controlled, but that HR changes as a function of consummatory activity. It is reasonable to question whether the deceleration $\mathrm{HR}$ response to dehydration found by Hufford and Chamber and by O'Kelly et al following thirst induced by hypertonic saline loads might be a consistent phenomenon in any given situation, and whether HR changes are a function of consummatory activity. If the deceleration $H R$ response to dehydration were a consistent phenomenon, then HR would decelerate as a function of deprivation in a given test situation where confounding factors are well controlled. Furthermore, if Eisman's hypothesis were valid, then HR would not vary as a function of deprivation in a given situation in which the rats are not required to produce bar-pressing responses for water or food.

Method. Three groups of 18 male naive hooded rats, about 90 days old at the beginning of this experiment, were assigned to three different conditions, i.e., six rats for the water-deprivation group, six for the food-deprivation group, and the remaining six for the control group. Prior to the experiment, three groups of rats had free access to both water and food. Then two experimental groups were deprived for $96 \mathrm{~h}$. During this period, the water-deprivation group always had food available and the food-deprivation group always had water available. For both groups, HR was recorded at $8 \mathrm{~h}$, $24 \mathrm{~h}, 48 \mathrm{~h}, 72 \mathrm{~h}$, and $96 \mathrm{~h}$ of deprivation by the Brush Electronics Company's Type B1-202 recorder via a Type 122 low-level preamplifier and a Model BL-91 3 DC amplifier.

The experimental apparatus was a simple rectangular wooden box, 2.5 in. wide $\times 3.0 \mathrm{in}$. high $\times 9 \mathrm{in}$. long inside, with a floor consisting of two electrically conductive parallel bars, $1 / 4 \mathrm{in}$. in diameter with a $3 / 4 \mathrm{in}$. gap between the two bars. The HR measurements were taken through the rats' paws. The box was placed in a shielded room.
In order to control confounding factors (e.g., handling, stimulus conditions, etc.), three weeks of adaptation trials ( $15 \mathrm{~min}$ recording of HR daily in the experimental box) were given for all rats prior to the beginning of the test trial proper. The test trial proper consisted of a 10 -min recording of $\mathrm{HR}$ after $3 \mathrm{~min}$ of adaptation in the box. Since the Ss showed greater locomotor activities immediately after they were placed in the test box, the mean HR counted from the last 3-min period was used for our data analysis.

Results. The relationship between HR changes and the hours of deprivation is summarized in Fig. 1. The group difference based on the mean HR was significant beyond the .05 level $(\mathrm{F}=5.098, \mathrm{df}=$ $2 / 90$ ). The treatment comparison between the control group and the experimental groups, i.e., both the water- and the fooddeprivation groups, showed a significant difference beyond the .01 level $(\mathrm{F}=10.193, \mathrm{df}=1 / 90)$. The treatment comparison between the food-deprivation group and the water-deprivation group, however, showed no significant difference.

The water-deprivation group showed that the HR changes and the hours of deprivation are monotonically related. The HR change over the deprivation time was significant beyond the .01 level $(F=7.65, d f=5 / 30)$. An orthogonal polynomials analysis showed that the HR change was linear within the range of the duration of deprivation applied. The linear trend was significant beyond the .05 level $(\mathrm{F}=4.18, \mathrm{df}=1 / 30)$.

The data for the food-deprivation group, however, showed a nonmonotonic relationship between the HR changes and the hours of deprivation. There was an increasing monotonic relationship between the 2- up to the 48-h deprivation, but after that the HR decreases sharply as the hours of deprivation increase. This change was significant beyond the .01 level $(F=8.03$, df $=5 / 30)$. The orthogonal polynomials analysis showed that the function was significantly quadratic beyond the .001 level $(\mathrm{F}=19.20, \mathrm{df}=$ $1 / 30$ ).

The total body weight changes as a function of the deprivation time were also recorded. Both experimental groups showed very similar trends up to $48 \mathrm{~h}$ of deprivation, then the total body

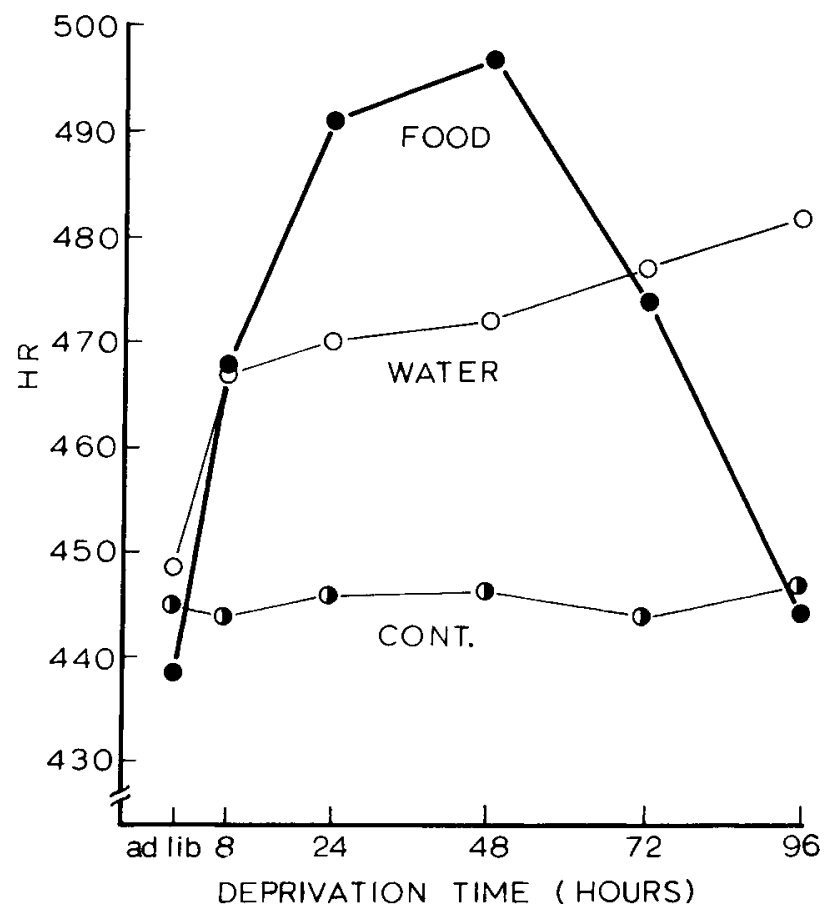

Fig. 1. Mean heart rate changes as a function of deprivation time. 
weight loss of the water-deprivation group became more severe than that of the food-deprivation group. But the difference was not significant $(F=1.808, \mathrm{df}=1 / 10)$. The loss of body weight was significant beyond the .05 level $(\mathrm{F}$-water $=7.05, \mathrm{df}=5 / 30$; and F-food $=5.53, \mathrm{df}=5 / 30$ ).

Discussion. The results obtained from the water-deprivation group in this experiment are in agreement with Bélanger \& Feldman's findings (1962) showing a positive linear function. Although the results of the waterdeprivation group support Bélanger and Feldman's findings, one should note that the experimental conditions of our study are not the same as theirs. In their study, HR was recorded while their rats were performing bar-press responses to obtain water. The number of bar-presses was also recorded. The data shows that the HR kept increasing while the response rate decreased after about $60 \mathrm{~h}$ of water deprivation. There was no isomorphic relationship between the HR and the number of bar-press responses. Bélanger and Feldman conclude, therefore, that HR is a function of water deprivation and that it is not a function of locomotor activities. Eisman (1966), however, argues that "Bélanger and Feldman's study reflects the effect of consummatory activity, rather than the effect of deprivation." In order to control the effect of consummatory activity, in our study, the HR was recorded in a well adapted nonhome cage in which the rats were not required to produce bar-pressing responses for water. Thus, the independent variable of the obtained linear function is clearly the duration of water deprivation. Our result, therefore, does not support Ducharme's (1966) statement that "... depriving an animal of water cannot per se produce a direct physiological effect leading to an increase in HR... [p. 103]."

The results obtained from the food-deprivation group do not agree with a linear function hypothesis. They show an inverse U-shaped function. The results also do not agree with Moller's findings, reported in his unpublished Master's thesis at McGill University in 1962. He obtained a significant reduction in HR as a function of food deprivation over a range of $6-46 \mathrm{~h}$. The differences between these two results may arise from the different experimental conditions. However, in the present study, since the Ss were tested in a well adapted nonhome cage and since no operant activity was required to obtain food in the cage, we can conclude that the direct independent variable for an inverse $\mathrm{U}$-shaped function is the duration of food deprivation. Thus the function for the food-deprivation group is clearly different from the function for the water-deprivation group.

It is puzzling, however, that the results of the food-deprivation group are quite different from those of the water-deprivation group. If dehydration as such causes tachycardiac change, then for the food-deprivation group a positive linear function should also be obtained, because the amount of water consumption of the rats during the food deprivation period steadily decreased down to a very small quantity. O'Kelly, Hatton, Tucker, \& Westall (1965) report that there is an inverse relationship between dehydration and HR, and that a bradycardiac trend is independent of the route of water loss whether produced by deprivation or osmotic transfer and that a tachycardiac trend is also "independent of the means by which $S$ acquires the water [p. 146]." The study by Hufford and Chambers also suggests that dehydration as such does not seem to be accompanied by tachycardiac change. These studies suggest that tachycardiac change may be affected by some other biological conditions than dehydration.

The results of the present study, however, seem to disagree with a conclusion drawn by O'Kelly et al (1965) and Ducharme (1966), and suggest that the HR is influenced by at least two concomitant conditions: dehydration and metabolic rate. For the water-deprivation group in our experiment, the amount of food consumption sharply decreased as the duration of water deprivation increased and the amount of food intake was almost null at the fourth day of deprivation. Thus, for this group, dehydration would have dominated to determine the physiological condition of the rats. Hence, a positive linear function of HR would be expected. For the food-deprivation group, however, although the amount of water intake decreased sharply, all rats consumed some amount of water. Thus, for this group the degree of dehydration is not so severe as that for the water-deprivation group, especially during the latter part of the deprivation period. The total body weight loss of this group, comparing with that of the water-deprivation group, also supports this assumption. This means that the effects of dehydration would dominate during the early part of the food deprivation period, then the different degree of dehydration and the effect of change in metabolic rate would gradually influence physiological processes. Thus, an inverse U-shaped function would be expected. These hypothetical statements require further research.

\section{REFERENCES}

BELANGER, D., \& FELDMAN, S. M. Effects of water deprivation upon heart rate and instrumental activity in rats. J. comp. physiol. Psychol, $1962,55,220-225$.

DUCHARME, R. Effect of internal and external cues on the heart rate of the rat. Canad. J. Psychol., 1966, 20, 97-104.

EISMAN, E. Effects of deprivation and consummatory activity on heart rate. J. comp. physiol. Psychol., 1966, 62, 71-75.

HAHN, W. W., STERN, J. A., \& McDONALD, D. G. Effects of water deprivation and bar pressing activity on heart rate of the male albino rat. $J$. comp. physiol. Psychol., 1962, 55, 786-790.

HULL, C. L. A behavior system. Yale Univ. Press, 1952.

MALMO, R. B. Activation. In A. J. Bachrach (Ed.), Experimental foundation of clinical psychology, New York: Basic Books, Inc. (1962 ed.).

O'KELLY, L. I., HATTON, G. I., TUCKER, L., \& WESTALL, D. Water regulation in the rat: Heart rate as a function of hydration, anestesia, and association with reinforcement. J. comp. physiol. Psychol, 1965, 59, 159-165. 